\title{
Effect of volatile compounds produced by Ralstonia solanacearum on plant growth promoting and systemic resistance inducing potential of Bacillus volatiles
}

Hafiz Abdul Samad Tahir ${ }^{1,2}$, Qin Gu', Huijun Wu', Waseem Raza', Asma Safdar ${ }^{1}$, Ziyang Huang ${ }^{1}$, Faheem Uddin Rajer ${ }^{1}$ and Xuewen Gao ${ }^{1 *}$

\begin{abstract}
Background: Microbial volatiles play an expedient role in the agricultural ecological system by enhancing plant growth and inducing systemic resistance against plant pathogens, without causing hazardous effects on the environment. To explore the effects of VOCs of Ralstonia solanacearum TBBS1 (Rs) on tobacco plant growth and on plant growth promoting efficiency of VOCs produced by Bacillus subtilis SYST2, experiments were conducted both in vitro and in planta.

Results: The VOCs produced by SYST2 significantly enhanced the plant growth and induced the systemic resistance (ISR) against wilt pathogen Rs in all experiments. The SYST2-VOCs significantly increased PPO and PAL activity and over-expressed the genes relating to expansin, wilt resistance, and plant defense while repressed the genes relating to ethylene production. More interestingly, VOCs produced by pathogen, Rs had no significant effect on plant growth; however, Rs-VOCs decreased the growth promoting potential of SYST2-VOCs when plants were exposed to VOCs produced by both SYST2 and Rs. The co-culture of SYST2 and Rs revealed that they inhibited the growth of each other; however, the inhibition of Rs by SYST2-VOCs appeared to be greater than that of SYST2 by Rs-VOCs.

Conclusion: Our findings provide new insights regarding the interaction among SYST2-VOCs, Rs-VOCs and plant, resulting in growth promotion and induced systemic resistance against the bacterial wilt pathogen $R s$. This is the first report of the effect of VOCs produced by pathogenic microorganism on plant growth and on plant growthpromoting and systemic resistance-inducing potential of PGPR strain SYST2.
\end{abstract}

Keywords: Bacillus subtilis, Growth promotion, Induced resistance, PPO and PAL, VOCs

\section{Background}

Bacterial wilt is a devastating and destructive soil born disease covering a range of nearly 450 crop species, particularly the crops of Solanaceae family [1, 2]. Bacterial wilt widely occurs in tropical and subtropical regions of the world [3]. The biological control method is environment-friendly, cost-effective and easily applicable method for the management of soil born plant

\footnotetext{
* Correspondence: gaoxw@njau.edu.cn

'Department of Plant Pathology, College of Plant Protection, Nanjing

Agricultural University, Key Laboratory of Integrated Management of Crop

Diseases and Pests, Ministry of Education, Weigang No.1, Nanjing 210095,

People's Republic of China

Full list of author information is available at the end of the article
}

pathogens. Plant growth promoting bacteria are not only involved in plant growth promotion but are also considered to be the best biocontrol agents, having the potential to suppress the population of pathogenic microorganisms and to induce the systemic resistance in plants against diseases [4]. The mechanisms involved in plant growth promotion comprising nitrogen fixation, secretion of phytohormones, solubilization of minerals [5-7], and production of antimicrobial compounds [8], including volatile organic compounds (VOCs) [9-12]. The VOCs produced by plant growth-promoting rhizobacteria (PGPR) are low molecular weight, gaseous, metabolic compounds emitted from bacterial cells 
under normal conditions [13], which are active even at low concentrations [14]. The VOCs produced by PGPRs play an expedient role in three ways; by controlling plant pathogens, stimulating plant growth and inducing systemic resistance [12, 15-18]. The mode of action of VOCs has an extra degree of advantage over other biocontrol and growth-regulating mechanisms that VOCs don't need any physical contact with pathogen or plant parts while most of the other processes involved in controlling phytopathogens and promoting plant growth, require physical contact and close vicinity $[4,5]$. Lemfack (2014) reported 300 bacteria and fungi as VOC producers, while 846 VOCs with 5431 synonyms were recorded in the database of volatiles emitted by microorganisms (DOVE-MO) [19].

Numerous bacterial species have been reported to have an influence on plant growth promotion and induction in systemic resistance including Bacillus, Pseudomonas, Stenotrophomonas, Serratia and Arthrobacter [15, 16, 20, 21]. Bacillus subtilis GB03 and B. amyloliquefaciens IN937a were reported first time by Ryu (2003) as the producer of plant growth promoting VOCs; 2,3-butanediol and acetoin [15]. A VOC 2pentylfuran emitted by Bacillus megaterium XTBG34 stimulated Arabidopsis thaliana growth [16] while 13tetradecadien-1-ol, 2-butanone and 2-methyl-n-1-tridecene, produced by Pseudomonas fluorescens SS101 promoted the growth of Nicotiana tabacum [18]. Besides their growth-promoting activity, the VOCs elicit plant tolerance against both biotic and abiotic elements by inducing systemic resistance. The bacillus VOCs, 2,3-butanediol have been testified to significantly induce resistance in the Arabidopsis plant against the pathogen Erwinia carotovora sub sp. carotovora [22] while 3-pentanol and 2-butanone, against the pathogen Pseudomonas syringae pv. lachrymans [23], a causal agent of bacterial angular leaf spot of cucumber. Similarly, another study reported that Paenibacillus polymyxa E681 produced tridecane, which significantly induced systemic resistance in plants [17].

A few studies have been reported concerning the mechanism of plant growth promotion and systemic resistance induction triggered by bacterial VOCs. Some researchers publicized that bacterial VOCs can interact with plant hormones by involving in morphogenetic processes, consequently trigger the plant growth promotion $[11,15,24,25]$. Xie et al. (2009) reported an enhancement in photosynthetic activity and chlorophyll contents, when Arabidopsis seedlings were exposed to VOCs of Bacillus subtilis GBO3 [26]. Transcriptional analysis of Arabidopsis after exposure to Bacillus subtilis GBO3-VOCs revealed that VOCs regulated the auxin which resulted in the initiation of growth promotion [24]. Furthermore, VOCs differentially expressed the transcriptional expression of genes relating to ethylene response and ethylene biosynthesis [27].

Among all PGPR species, Bacillus species are deliberated as the most efficacious species since they have the capability to produce spores that can persist in adversative environmental conditions [28]. In our previous studies, we identified two VOCs, albuterol and 1,3propanediole produced by Bacillus subtilis SYST2 that promoted the plant growth of tomato [11]. The main objective of this study was to explore the impact of VOCs produced by SYST2, in-vitro and in-planta, on plant growth promotion and activation of induced systemic resistance in tobacco; in addition, the effect of VOCs produced by pathogen Ralstonia solanacearum on the activity of VOCs of PGPR strain SYST2 was also explored.

\section{Results}

SYST2-VOCs enhance tobacco plant growth while Rs-VOCs decrease the efficiency of PGPR strain SYST2 In vitro assay

To determine the effect of SYST2-VOCs on plant growth in vitro, tobacco seedlings were grown in divided Petri plates using MS medium for plant growth, and in "two plate system" using soil as a medium for plant growth. In divided plate system, data were taken after 14 days and a significant increase was observed in fresh green weight and dry weight of tobacco plants exposed to SYST2-VOCs as compared to the water control and DH5 $\alpha$ control. The VOCs emitted by SYST2 enhanced both the fresh and dry weights of tobacco plants significantly, by 7.7-fold, compared to the controls (Fig. 1a and b). Similarly, in two plate system, the SYST2-VOCs significantly enhanced both fresh and dry weights of tobacco plants by 3.5 fold, compared to controls (Fig. 1C1$1 E)$. No significant differences were noted when the results of plants exposed to the VOCs of E. coli DH5 $\alpha$ (negative control) were compared to the water control in both experimental systems (Fig. 1).

To examine whether Rs-VOCs also affected plant growth or affected the efficiency of PGPR strain SYST2, we created a "three partitioned plate system" using MS medium for plant growth. In one partition tobacco seedlings were grown while in other two partitions, PGPR strain SYST2 or pathogenic strain Rs was inoculated. Our results revealed that $R s$-VOCs had no effect on plant growth and showed similar results compared to control. However, the plates in which both SYST2 and $R s$ were inoculated in two separate partitions, the growth promoting effect of SYST2-VOCs was significantly lower, compared to the treatment where tobacco seedlings were exposed to SYST2-VOCs only. Rs-VOCs significantly decreased the growth-promoting potential of SYST2. Almost a 7-fold increase was observed when 

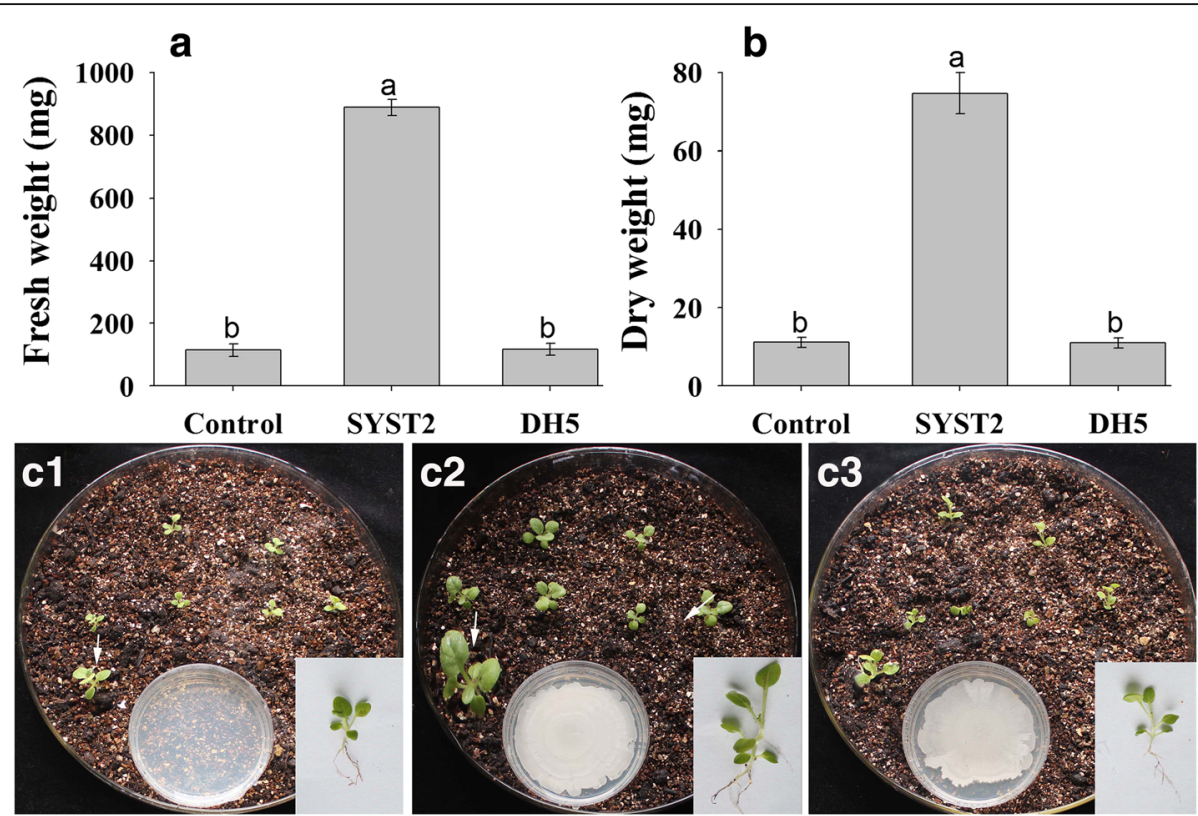

d
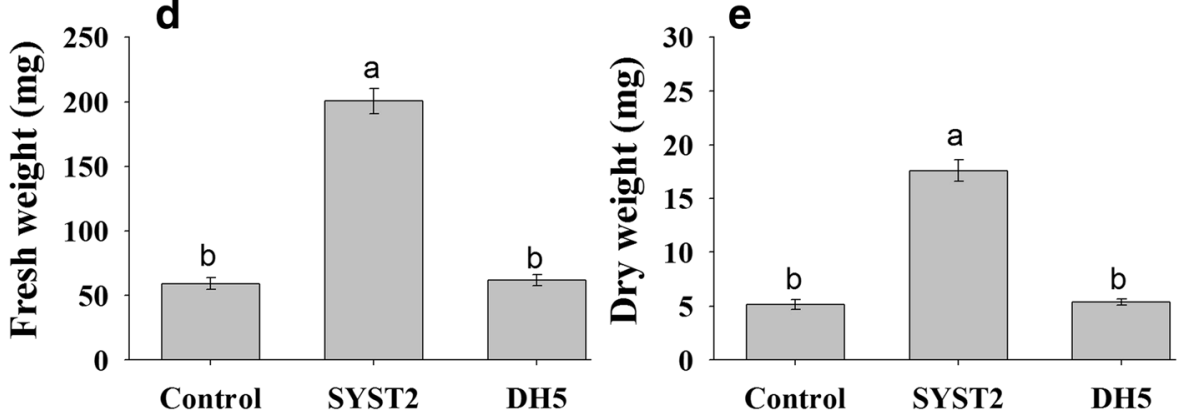

Fig. 1 Effect of SYST2-VOCS on Plant growth promotion of tobacco in vitro. For divided plate system (a and $\mathbf{b})$, seven germinated seedlings were transplanted in one partition and bacteria were spot inoculated on the other partition. For "two plate system", germinated seedlings were grown in larger plates having soil mixed with vermiculite as a medium for growth, while bacterial strains were inoculated in small plates. The effect of SYST2-VOCS on plant growth was observed by recording fresh green weight (a) and dry weight (b) after two weeks of exposure. In "two plate system" effect was observed visually which showed different growth in control (C1), exposed to SYST2-VOCs (C2), and exposed to DH5-VOCs (C3). Fresh weight of tobacco seedlings (d) and dry weight (e) of seedlings were recorded after 10 days in "two plate system". Error bars indicate the standard deviation of the mean $(n=5)$. Different lower case letters above the columns represent significant differences between treatments at $P=0.05$. Experiments were repeated three times with similar results

tobacco seedlings were exposed to SYST2-VOCs only while a 4.5 -fold increase was observed when exposed to SYST2-VOCs accompanied with Rs-VOCs (Fig. 2).

\section{In planta assay}

To investigate the plant growth-promoting potential of VOCs in planta, pot experiments was conducted as described by Park et al. [18]. Five tobacco seedlings were transplanted into pots attached to the inside of jars containing a culture of SYST2 or DH5 $\alpha$ (for control) at the bottom. After 28 days exposure, the analysis of the data showed a significant increase in the growth of tobacco seedlings in terms of fresh green and dry weight, and leaf area as compared to the water and DH $5 \alpha$ controls (Fig. 3a-3h). A significant increase of almost 1.6-fold in photosynthesis rate was also observed after exposure to SYST2-VOCs. Based on our data, we can state that the growth and development of tobacco seedlings was stimulated by B. subtilis SYST2 VOCs in all experimental systems. In this experiment, in addition to the evaluation of growth promoting activity of VOCs by SYST2, we evaluated the effect of $R s$-VOCs on plant growth regulation and on the efficiency of PGPR strain SYST2 in planta. For this purpose, in one set we inoculated only $R s$ or SYST2 while in other we used two plates; one inoculated with SYST2 and one inoculated with Rs. RsVOCs exhibited no effect on plant growth regulation and showed similar results, compared to control. Tobacco seedlings exposed to a combination of both SYST2 and Rs had a lesser effect on plant growth 


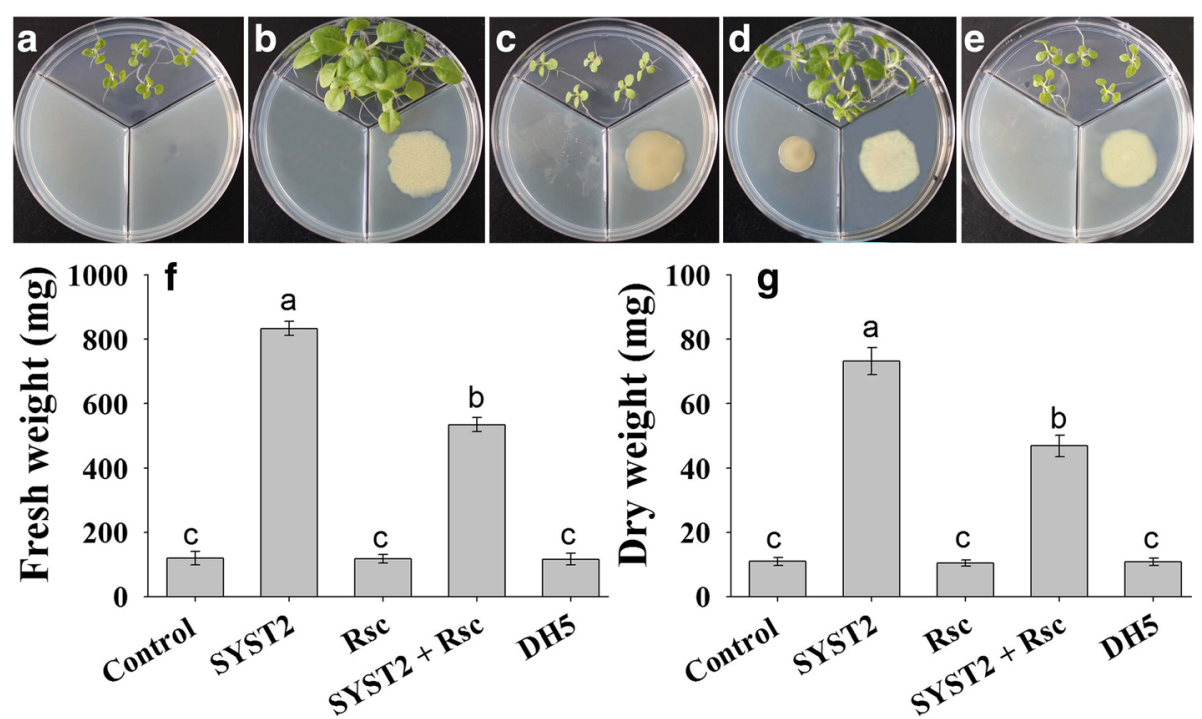

Fig. 2 Effect of Rs-VOCs on plant growth promoting potential of SYST2-VOCs in vitro. Three partitioned plate system was used to evaluate the effect of Rs-VOCs on growth-promoting potential of SYST2-VOCs. After two weeks of exposure to VOCs, observations were recorded; control (a), SYST2 only (b), Rs only, (c) SYST2 and Rs together (d) and DH5a (e) as a negative control. Fresh (f) and dry weights (g) of tobacco seedlings were recorded. Error bars indicate the standard deviation of the mean $(n=5)$. Different lower case letters above the columns represent significant differences between treatments at $P=0.05$. Experiments were repeated three times with similar results

promotion, compared to only SYST2-VOCs in terms of all studied parameters; fresh and dry weight, leaf area and photosynthesis rate. A 2.5 fold increase was observed in fresh and dry weight, 1.84 in leaf area and 1.53 in photosynthesis rate when exposed to SYST2-VOCs only while the values were decreased to 2 -fold for fresh and dry weight and 1.25-fold both for leaf area and photosynthesis when exposed to SYST2-VOCs accompanied with Rs-VOCs (Fig. 3).

\section{Plant growth promoting activity by specific VOCs produced by SYST2}

All of the compounds produced by SYST2 were tested individually to evaluate their plant growth promoting potential. The two compounds, albuterol and 1,3-propanediol, positively stimulated plant growth promotion as compared to the control. Five germinated tobacco seedlings of equal size were transferred to the plastic pots, fixed on the glass jars while individual compounds were dissolved in DMSO and applied to the plates, which were placed at the bottom of the jar, and incubated at $28 / 22{ }^{\circ} \mathrm{C}$ day/night under $16 / 8$-h light/dark photoperiod for 14 days. The results revealed that albuterol $(10 \mathrm{mM}$ \& $1 \mathrm{mM})$ while 1,3 -propanediol $(100 \mathrm{mM} \& 10 \mathrm{mM})$ promoted the growth significantly, compared to control. However, no difference was observed when seedlings were exposed to $0.1 \mathrm{mM}$ albuterol and $1 \mathrm{mM}$ 1,3-propanediole, compared to control. A significant difference was observed in fresh green weight, dry weight, leaf area and photosynthesis rate in tobacco plants after exposure to these volatile compounds. Albuterol $(1 \mathrm{mM})$ increased fresh green and dry weight by 2.14 -fold, while 1,3-propanediol $(10 \mathrm{mM})$ enhanced fresh green weight by 1.76 -fold and dry weight by 1.61 -fold as compared to the control (Fig. 4).

\section{Effect of VOCs produced by Rs on the growth of PGPR strain SYST2}

The VOCs produced by $R s$ reduced the growthpromoting potential of VOCs derived by PGPR strain SYST2 as described in the above experiment. To investigate interaction among VOCs produced by PGPR strain SYST2 and pathogenic strain $R s$, we examined the effect of Rs-VOCs on the growth of SYST2 and vice versa. Results showed that SYST2-VOCs inhibited the colony growth of $R s$ while Rs-VOCs inhibited the growth of SYST2; however, this inhibition level was different for SYST2 and Rs against each other. SYST2-VOCs inhibited the colony growth of Rs more compared to the inhibition of SYST2 growth by Rs-VOCs. SYST2-VOCs inhibited the growth of $R s$ up to $54 \%$, but $R s$-VOCs inhibited the growth of SYST2 up to $22 \%$. VOCs produced by SYST2 or Rs have no effect on their own colony growth when cultured same bacteria on both sides of the I-plate (Fig. 5a and b).

To determine which VOCs produced by Rs, we collected VOCs, by a combination of HS-SPME and GCMS. Twelve compounds were identified from Rs (see Additional file 1, Table S2), which had relatively high peak areas, e.g., $\geq 1 \%$, and were not similar to the 

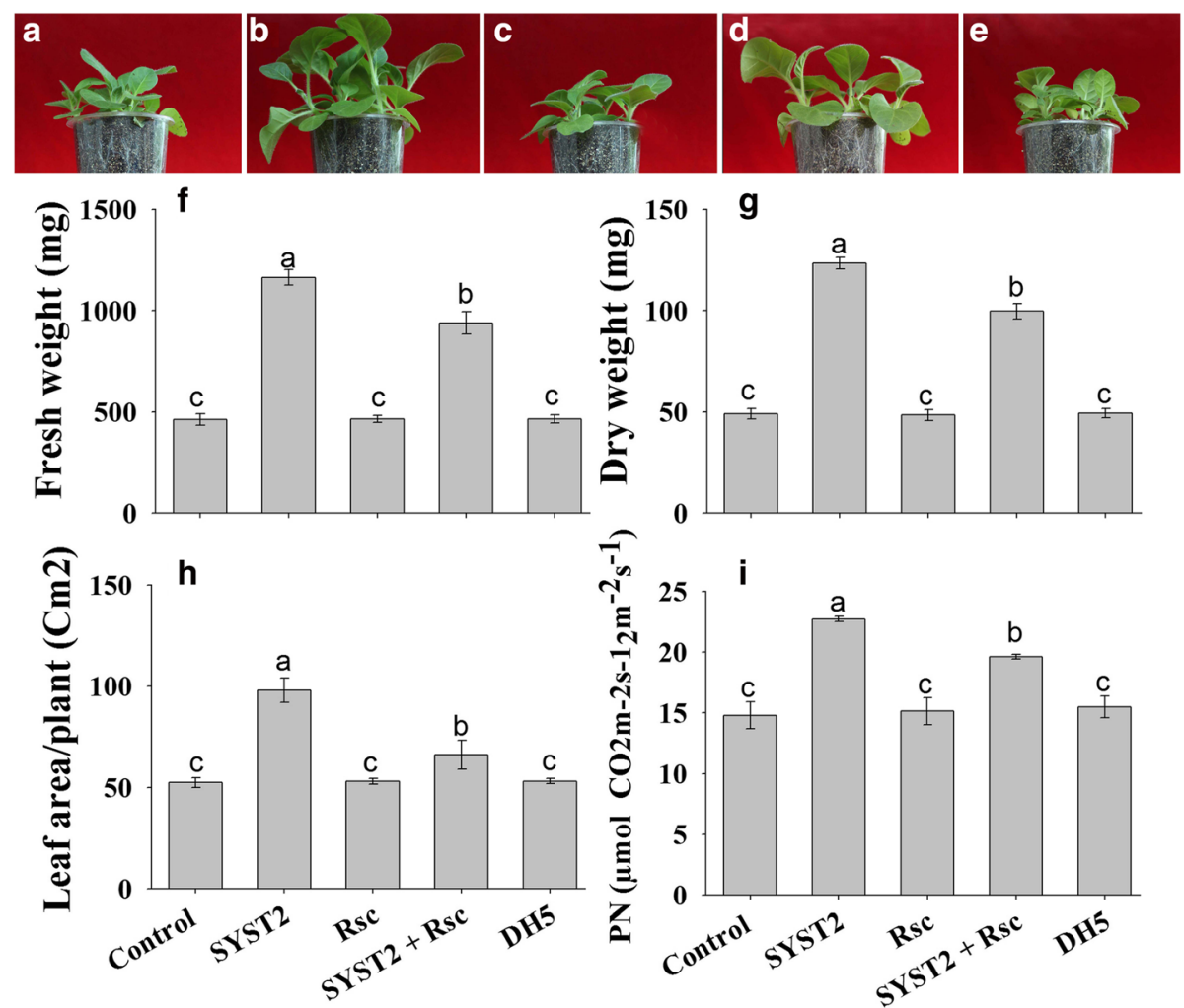

Fig. 3 Effect of Rs-VOCS on plant growth promoting potential of SYST2-VOCs in planta. Five germinated seedlings were grown in pots attached to the inside of jars containing a culture of SYST2, Rs or both together. Pots were placed in a growth chamber at $28 / 22^{\circ} \mathrm{C}$ day/night under $16 / 8$-h light/dark photoperiod. A clear difference was observed in control (a) and after 28 days of exposure to SYST2-VOCs alone (b), Rs-VOCS (c), SYST2 and Rs-VOCs together (d), and DH5a (e). Fresh green weight (f), dry weight $(\mathbf{g})$, leaf area (h), and photosynthesis rate (i) was recorded after 28 days. Error bars indicate the standard deviation of the mean $(n=5)$. Different lower case letters above the columns represent significant differences between treatments at $P=0.05$. Experiments were repeated three times with similar results

control. Dichloroacetic acid, 2-ethylhexyl ester (DCA), 2-tetradecanone (2-TD) and butanamide had antibacterial activity against SYST2 and inhibited the growth of SYST2 by $35.23 \%, 21.23 \%$, and $21.02 \%$, respectively (Fig. 5c).

\section{SYST2-VOCs reduced the wilt disease index by inducing systemic resistance}

SYST2-VOCs reduced the wilt disease development significantly (18.66\%) when tobacco seedlings were exposed to its VOCs, compared to the control (91.66\%), in two plate system. Similarly, individual VOCs albuterol and 1,3-propanediol also reduced the disease development. A disease index of $14.4 \%$ and $15.2 \%$ was observed by albuterol ( $1 \mathrm{mM}$ and $0.1 \mathrm{mM})$ while $18.4 \%$ and $20.8 \%$ by 1,3-propanediol (10 $\mathrm{mM}$ and $1 \mathrm{mM}$ ) compared to (97.6\%) water control. Reduction in disease development, both by SYST2-VOCs collectively, and by individual VOCs in vitro, revealed the mechanism of induced systemic resistance in tobacco against Rs by SYST2-VOCs as well as by individual synthetic chemicals (Fig. 6a-c). To demonstrate the effect of VOCs produced by SYST2 on induced systemic resistance at a broader level (in-vivo), we created the in planta system as described above. The results revealed that VOCs produced by SYST2 caused a significant reduction in the wilt index, confirming the induced systemic resistance in plants against Rs. In the pot experiment, SYST2 significantly reduced the disease and showed only a $33 \%$ wilt index, as compared with a $90.66 \%$ wilt index in the non-exposed control while albuterol $(1 \mathrm{mM})$ showed only $19 \%$ wilt index followed by 1,3-propanediol $(1 \mathrm{mM})$ and albuterol $(0.1 \mathrm{mM})$ with $27 \%$ wilt index (Fig. 6 d-f). No significant difference was observed in water control and when plants exposed to DH5 $\alpha$ (-ve control).

Evaluation of the activity of resistance-related enzymes. The effect of SYST2-VOCs alone and in combination with Rs-VOCs on the activity of enzymes, relating to resistance (PPO and PAL), was determined in response to inoculation of bacterial wilt pathogen $R s$ in plants that were exposed to VOCs. The activities of PPO and PAL were low and have no significant difference among VOCs-exposed and control plants before pathogen 
a
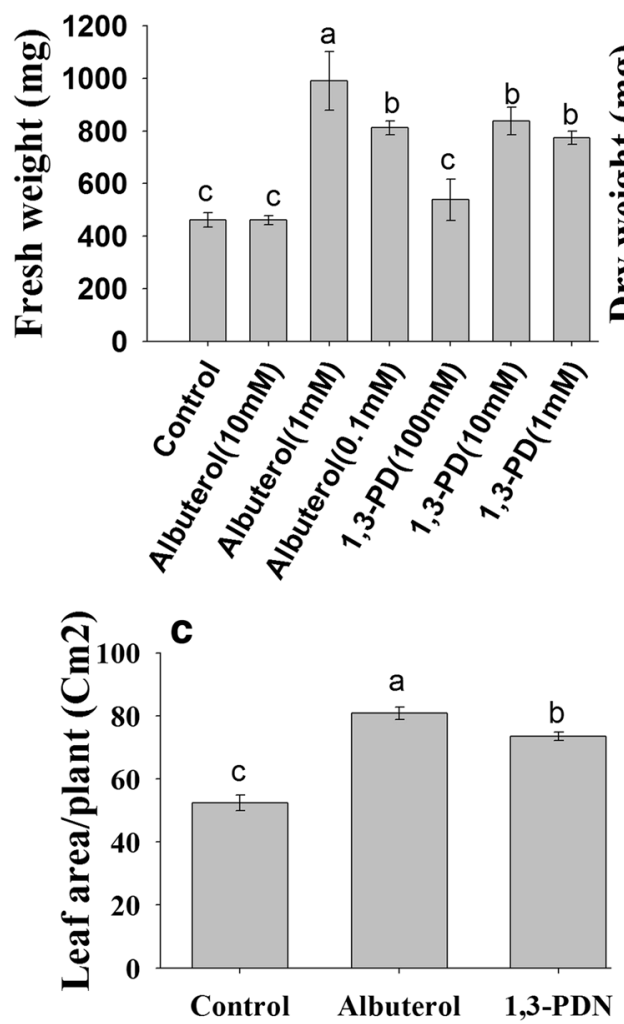

b
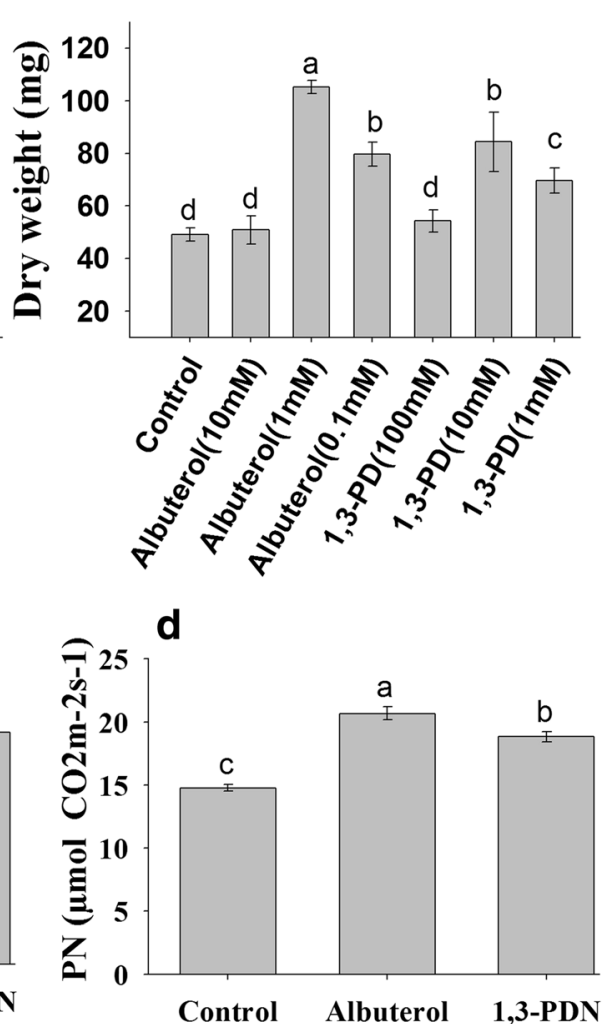

Fig. 4 Evaluation of individual VOCS produced by B. subtilis SYST2 for plant growth promotion. Tobacco seedlings were exposed to the specific individual VOCs albuterol and 1,3-propanediol dissolved in DMSO at four different concentrations in pots as described above for 28 days at $28 /$ $22^{\circ} \mathrm{C}$ day/night under 16/8-h light/dark photoperiod. Plant fresh weights (a), dry weights (b), leaf area (c) and photosynthesis rate (d) were determined. Error bars indicate the standard deviation from the mean. Letters above the columns indicate a significant difference at $P<0.05$. Experiments were replicated three times

inoculation. A clear increase was observed both in PPO and PAL after pathogen inoculation in plants that were exposed to VOCs when compared to both inoculated and non-inoculated control. The increase in enzyme activity was reached to its highest point at $48 \mathrm{~h}$ of inoculation and started to decline after $72 \mathrm{~h}$, reaching its previous position after $120 \mathrm{~h}$ of $R s$ inoculation. However, the activities of both the PPO and PAL enzymes were higher in plants exposed to SYST2-VOCs as compared to SYST2 + Rs-VOCs. No increase in enzyme activity was observed in non-inoculated control plants while no significant difference was observed between plants exposed to DH $5 \alpha$-VOCs compared to control (Fig 7).

\section{VOCs affect the transcription of genes related to plant growth regulation and resistance}

To investigate whether the induced systemic resistance and growth promoting activity of VOCs is related to alteration in the relative transcriptional expression of genes, we examined the transcriptional levels of some key genes related to expansin (NtEXPA1 and NtEXPA2), ethylene (ACO-1), resistance (RRS1) and pathogenesis- related proteins $(\operatorname{Pr} 1 a$ and $\operatorname{Pr} 1 b)$. Samples were taken at 7,14 , and 21 days after exposure to VOCs produced by SYST2, total RNA was extracted, and the firststrand cDNA was synthesized from the polyadenylated mRNA. qRT-PCR assays performed with gene-specific primers demonstrated that SYST2-VOCs changed the relative expression of all the tested genes to varying levels (Figs. 8 and 9). The relative expression of the two expansin genes NtEXPA1 and NtEXPA2 was clearly increased after exposure to SYST2-VOCs, compared to control. However, increase in expression level was less when plants were exposed to SYST2-VOCs along with Rs-VOCs. Our results showed a clear down-regulation of the ACO-1 gene after exposure to SYST2 VOCs alone or in combination with $R s$-VOCs, at varying levels, compared to the control. ACO-1 encodes ACC oxidase, a key enzyme that catalyzes the final step in ethylene biosynthesis. No significant effect was observed on the relative expression of these genes in water control and DH5 $\alpha$ control. Similarly, albuterol and 1,3-propanediol up-regulated both the $N t E X$ PA1 and NtEXPA2 while down-regulated the ACO-1 


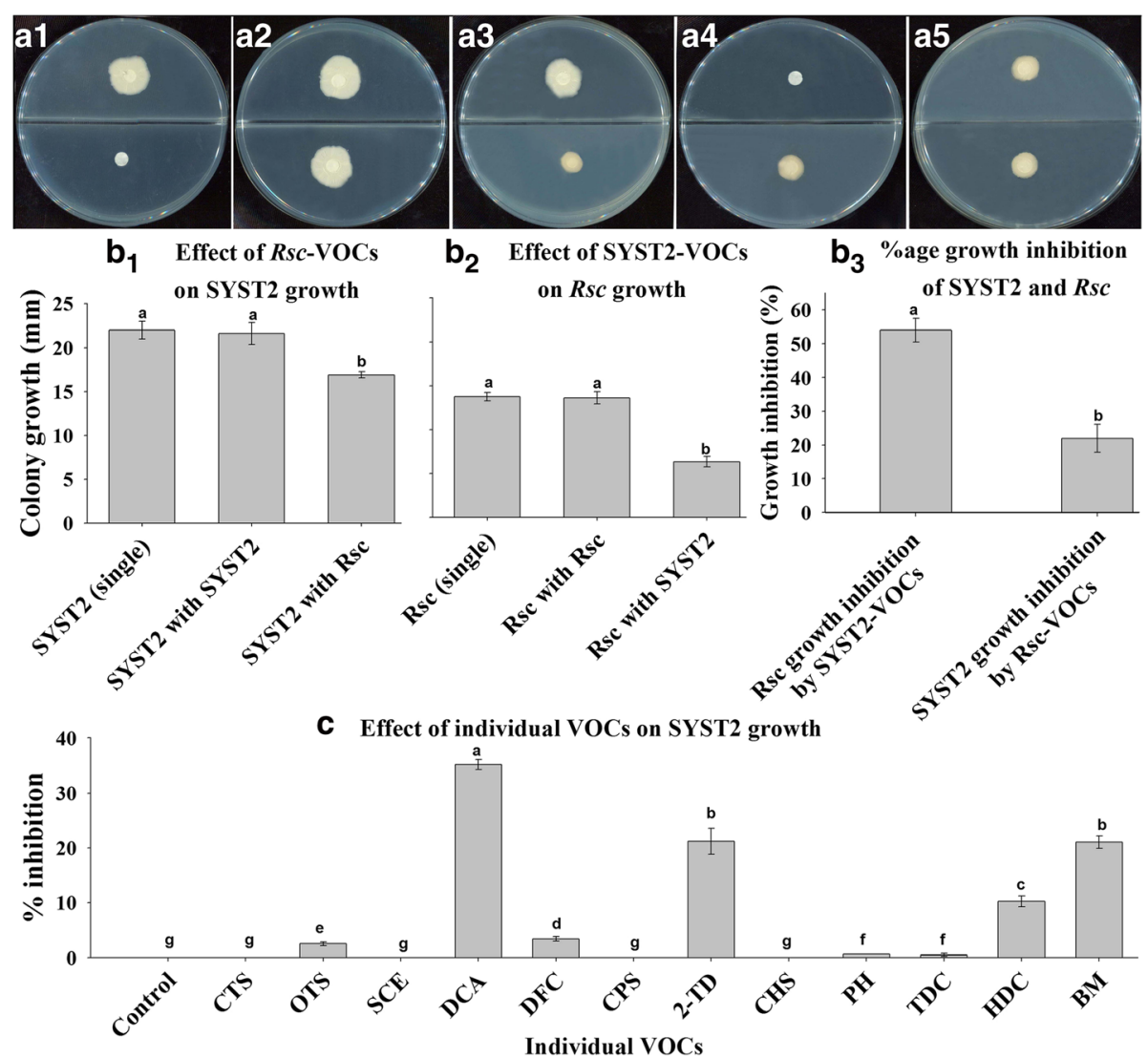

Fig. 5 Effect of VOCs produced by Rs on the growth of PGPR strain SYST2. Ten $\mu$ l of (18-24 h) Rs culture $\left(1 \times 10^{7}\right.$ CFU $\left.\mathrm{ml}^{-1}\right)$ grown in CPG broth was dropped in one partition of I-plate while $10 \mu \mathrm{l}$ of the liquid overnight-grown culture of SYST2 $\left(1 \times 10^{8} \mathrm{CFU} \mathrm{ml}^{-1}\right)$ was dropped in the other partition containing modified minimal salt medium (MS). The plates were double sealed and incubated at $28^{\circ} \mathrm{C}$ for three days. Only pathogen and only SYST2 were inoculated on control plates. The diameter of Rs and SYST2 were measured in mm. Only SYST2 (a1), SYST2 with SYST2 (a2), SYST2 with Rs (a3), only Rs (a4) and Rs with Rs (a5). Effect of VOCs produced by pathogenic strain Rs on SYST2 growth (b1) and SYST2-VOCS on Rs growth was observed (b2) and percentage growth inhibition (b3) was recorded. Similarly, the effect of specific VOCs produced by Rs was evaluated (c). Error bars indicate the standard deviation from the mean. Each experiment was performed with five replicates, and the experiment was repeated three times

(Figs. 8 and 9). Furthermore, we analyzed the genes of tobacco plant relating to resistance and defense by realtime PCR, to verify the resistance in tobacco against $R s$, due to the exposure of VOCs. The transcriptional expression of $\mathrm{R}$ gene $R R S 1$, involved specifically, in wilt resistance in tobacco against $R s$ was induced by the exposure to SYST2-VOCs and also by individual chemicals; albuterol and 1,3-propanediole compared to untreated control. However, over-expression was more by SYST2-VOCs as compared to individual chemicals and increased with the time, displaying its maximum at 9th days after inoculation. An up-regulation of defenserelated proteins Prla and Pr1b genes was also observed after exposure to SYST2-VOCs. However, there was the difference in the relative expression levels of Pr1a and Pr1b among the VOCs produced by SYST2 and specific individual VOCs. Increased expression of Prla and Pr1b was noticed when treated with SYST2-VOCs compared to individual VOCs, which reached highest at 9th days (Figs. 8 and 9). Our result revealed that VOCs stimulated the resistance and defense-related genes which resulted in the induction of systemic resistance in tobacco against $R$ s.

\section{Discussion}

Volatiles produced by microorganisms have been reported to have a valuable environment friendly role, in the form of beneficial interactions among PGPRs and plants, resulting in the induction of systemic resistance against biotic and abiotic elements [11, 17, 29], plant growth promotion $[18,23]$ and inhibition of plant fungal and bacterial pathogens [30]. Most of the previous studies on plant growth regulation and induced systemic resistance by bacterial VOCs described the interaction between VOCs produced by PGPR strain and plants only $[12,15,21,27]$. In this study, we clearly 

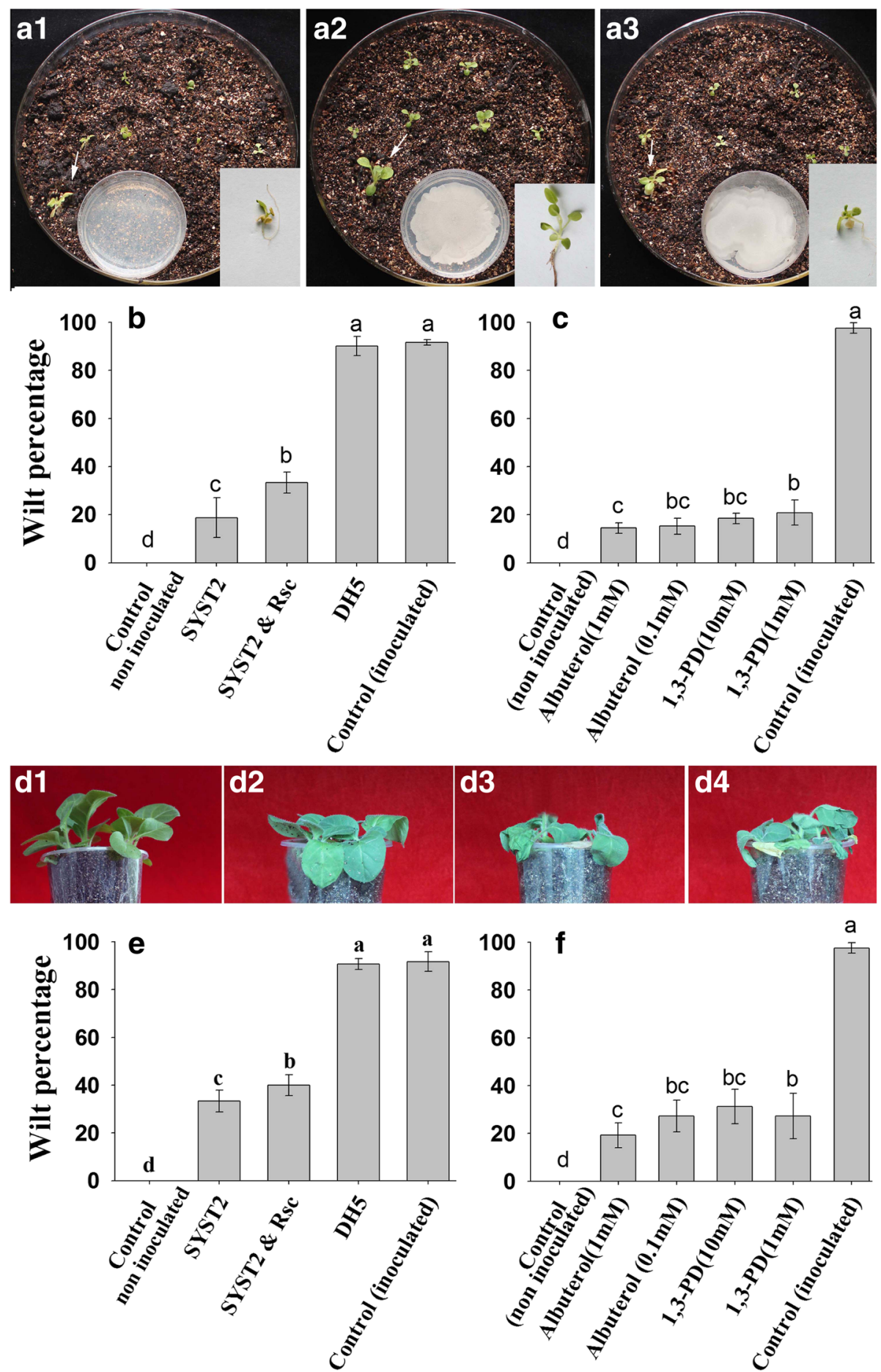

Fig. 6 SYST2-VOCs reduced the wilt disease index by inducing systemic resistance. For in vitro assay, germinated seedlings were dipped in Rs culture and grown in larger plates having soil mixed with vermiculite as a medium for growth. Sterilized water was used as control (a1) while PGPR strain SYST2 (a2) and DH5a (a3) were inoculated in small plates. Wilt index was recorded after 14 days of inoculation after exposure to SYST2-VOCs (b) and synthetic VOCs (c). For in planta assay, the pots were inoculated with a suspension of Rs (at an OD of 0.1 at $600 \mathrm{~nm}$ ) by dipping the roots in the suspension, except the non-inoculated control (d1), and then the seedlings were re-planted in the pots. Small agar plates at the bottom of jars were inoculated with SYST2 (d2), DH5a (d3), while only sterilized water was used as control (d4). Data was recorded after 21 days of inoculation after exposure to SYST2-VOCs (e) and synthetic VOCS (f) 


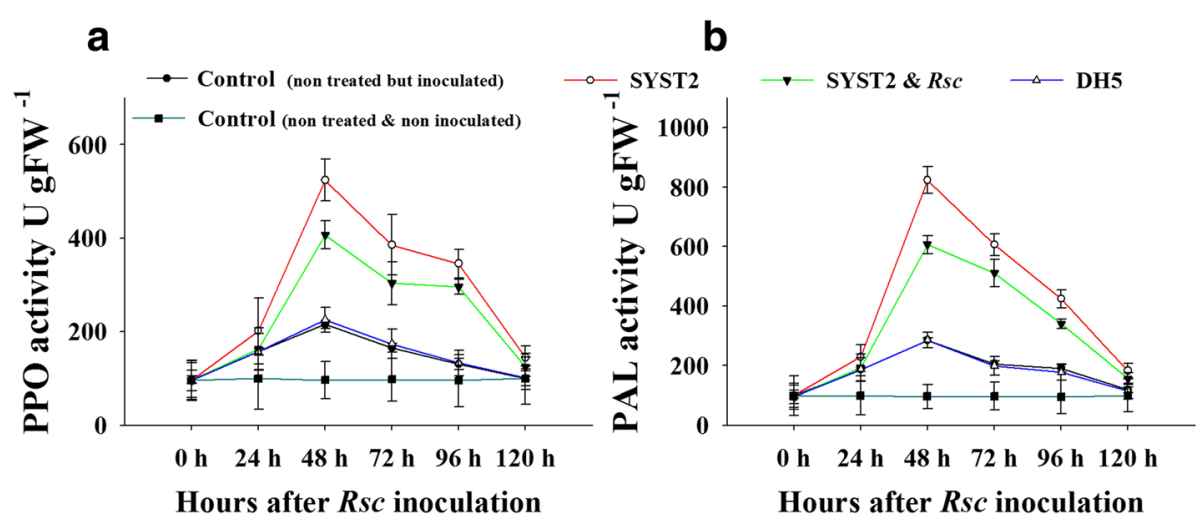

Fig. 7 Polyphenol Oxidase (PPO) and Phenylalanine Ammonia Lyases (PAL) Activity. Leaf samples were collected 0 (pre-inoculation), 24, 48, 72, 48, 72, 96 and $120 \mathrm{~h}$ after inoculation of Rs and activity of PPO (a) and PAL (b) was measured. Error bars indicate the standard deviation from the mean. Each experiment was performed with five replicates, and the experiment was repeated three times

demonstrated the interaction among PGPR strain Bacillus subtilis SYST2, plant pathogenic bacteria Ralstonia solanacearrum TBBS1 and tobacco plant. Both the beneficial and pathogenic bacterial strains coexist in soil and their efficiency as a PGPR or pathogen depends on their populations, nutrition and environmental conditions. In soil, many interactions coexist that affect each other's biological activity. In this research, we choose to study the interactive effects of volatile compounds (VOCs) produced by both PGPR strain and pathogen Rs. A significant difference was observed in all growth parameters (fresh weight, dry weight, and leaf area) when tobacco seedlings were exposed to only SYST2-VOCs or individual compounds, albuterol and 1,3-propanediol in all experimental systems. No significant difference was observed following exposure to VOCs produced by pathogenic bacteria Rs. However, Rs-VOCs decrease the growth promoting potential of SYST2-VOCs, as a clear difference was observed when tobacco seedlings were exposed to only SYST2-VOCs or SYST2-VOCs in combination with Rs-VOCs. This decrease in growthpromoting potential of SYST2-VOCs suggests the growth inhibiting effect of Rs-VOCs against PGPR strain SYST2. To determine the interaction between Rs-VOCs and SYST2-VOCs, we inoculated SYST2 and Rs alone, and in combination, using I-plate system. Both SYST2VOCs and Rs-VOCs inhibited the growth of each other but at different levels. However, SYST2-VOCs inhibited the growth of $R s$ more (56\%) as compared to Rs-VOCs which inhibited the growth of SYST2 up to $22 \%$ (Fig. 5), suggesting the possibility that Rs-VOCs might be involved in decreasing the growth-promoting potential of PGPR strain SYST2 by inhibiting its growth. Most prior studies used I-plate system for the evaluation of the effect of VOCs on plant growth promotion or induced systemic resistance. MS-media is being used for plant growth in I-plates and due to its solid or semi-solid nature; VOCs only can reach the leaves while roots remained unexposed. Another point is that when we inoculate pathogen to evaluate induced systemic resistance, pathogen grows over the surface of MS-media and contaminates the I-plate. In this study, we modified the method, using "two plate system" in which soil mixed with vermiculite was used as a medium for plant growth. In this system, VOCs could reach the roots of seedlings through small pores present in the soil-vermiculite mixture. A significant increase in fresh and dry weights of tobacco was observed in this system following exposure to SYST2-VOCs; however, this increase was less (3.5fold) as compared to I-plate system (7.7-fold). This difference might be due to unequal conditions for growth of seedlings in MS medium or soil medium, as MS medium is better for plant growth. Growth conditions in vitro and in planta are relatively different in terms of growing media and the degree of interaction of the tobacco with numerous environmental factors. PGPR live in soil naturally, and under natural conditions, the VOCs produced by PGPR usually interact with plant roots rather than leaves. Therefore, we moved the experiment from in vitro to pots in a growth chamber in order to determine the growth promoting activity by SYST2VOCs in soil to expose the roots to VOCs. Our results revealed that SYST2 VOCs stimulated the growth under soil conditions also, which support the results of Park et al. [18].

Volatile organic compounds produced by microorganisms induced several physiological alterations relating to growth hormones and photosynthesis [24]. Our results showed a significant increase in photosynthesis rate, following the exposure to SYST2-VOCs. Bacillus subtilis GBO3-VOCs have been reported to enhance photosynthetic rate by increasing endogenous content of chlorophyll [31] and by changing the transcription of chloroplasts related genes, suggesting a correlation 


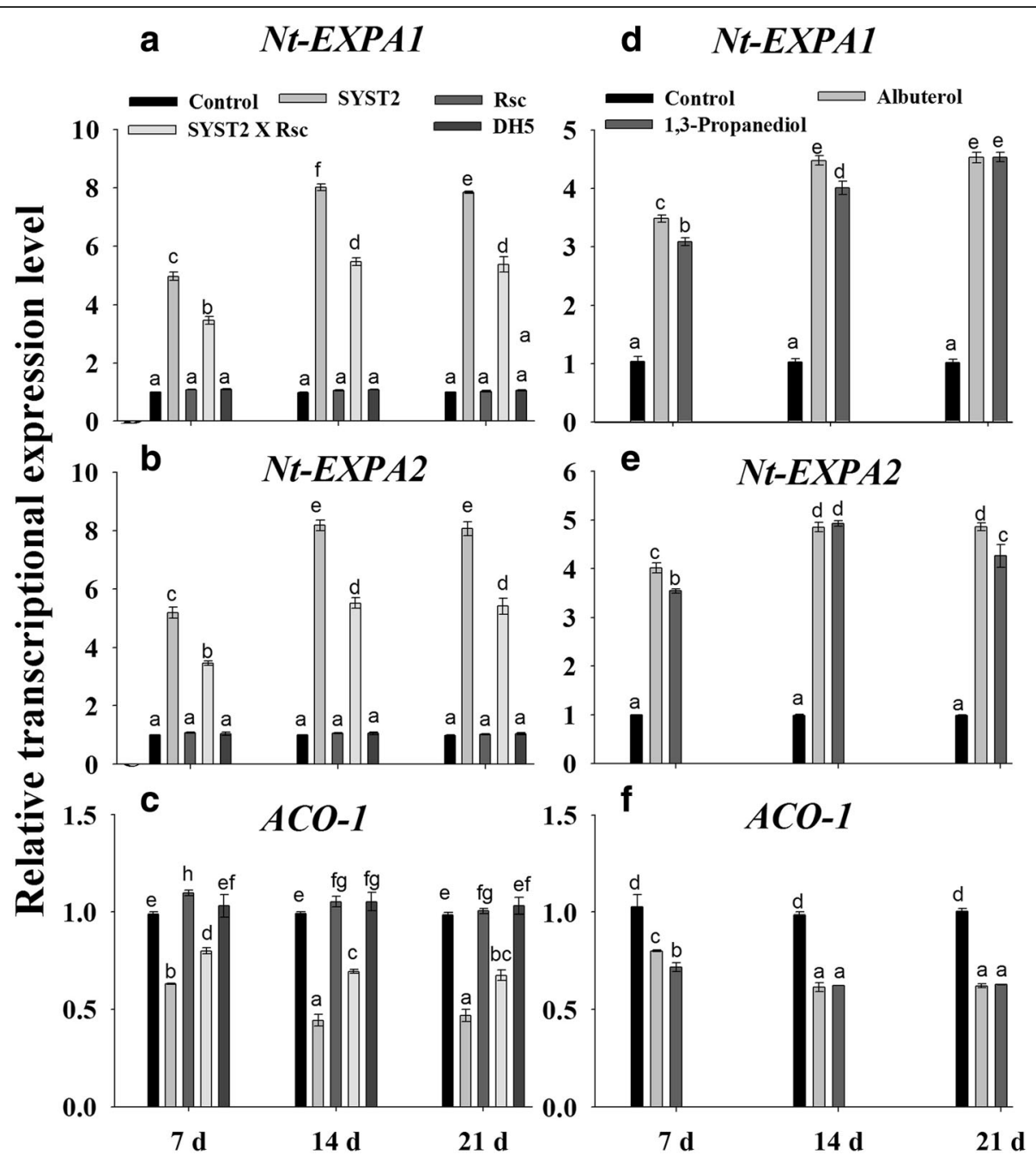

Fig. 8 Transcriptional expression profiles of genes involved in growth regulation after exposure to VOCs. QRT-PCR was performed with SYBR Green/Fluorescent qPCR master mix (Takara) on a Roche-480 system (Roche), and EF-1a was used as an internal reference. The relative expression levels of Nt-EXPA7(a), Nt-EXPA2 (b) and ACO-7(c) were determined after exposure to VOCs by SYST2 alone or in combination with Rs. Similarly, the relative expression levels of Nt-EXPA7(d), Nt-EXPA2 (e) and ACO-1 (f) were determined after exposure to synthetic VOCs. Error bars indicate standard errors of the means. Different lower case letters above the error bars represent significant differences according to Duncan's multiple-range test $(P \leq 0.05)$ using SPSS software (SPSS, Chicago, IL)

between the increase in photosynthetic activity and plant growth enhancement [32]. Up-regulation of transcriptional expression of the expansin genes EXPB1, EXPB3, EXP4, and EXP5 in Arabidopsis [24], EXP1, EXP2, and EXP6 in Nicotiana $[5,33]$, and EXPA5 in Lactuca sativa [34] resulted in cell expansion. Nt-EXPA1 and Nt-EXPA2 encode tobacco expansin proteins that stimulate cell division and extension by loosening cell walls while $A C O 1$ gene (1-aminocyclopropane-1-carboxylic acid oxidase) is an important enzyme which regulates the ethylene production [35]. Our results showed that SYST2 VOCs up-regulated the transcriptional expression of the expansin-related genes $N t$-EXPA1 and Nt-EXPA2 while a down-regulation was observed in ACO1. Our findings support formerly reported studies that bacterial volatiles has the potential to alter the transcriptional expression of genes relating to ethylene biosynthesis $(A C O 2, A C S 4$, $A C S 12$, and $S A M-2)$ and ethylene response (CHIB, ERF1, and GST1) [36]. Our results showed that SYST2VOCs or synthetic VOCs, albuterol and 1,3-propanediole negatively influenced the wilt disease development by activating the induced systemic resistance in tobacco plants against bacterial wilt pathogen $R$. solanacearum TBBS1. However, induced systemic resistance appeared to be greater under in vitro conditions as compared to in planta. The difference observed could be due to the larger volume of the glass jars resulting in a lower concentration and availability of VOCs to the seedlings in the pots. In our previous experiment, synthetic VOCs, albuterol and 1,3-propanediole have been proved to 


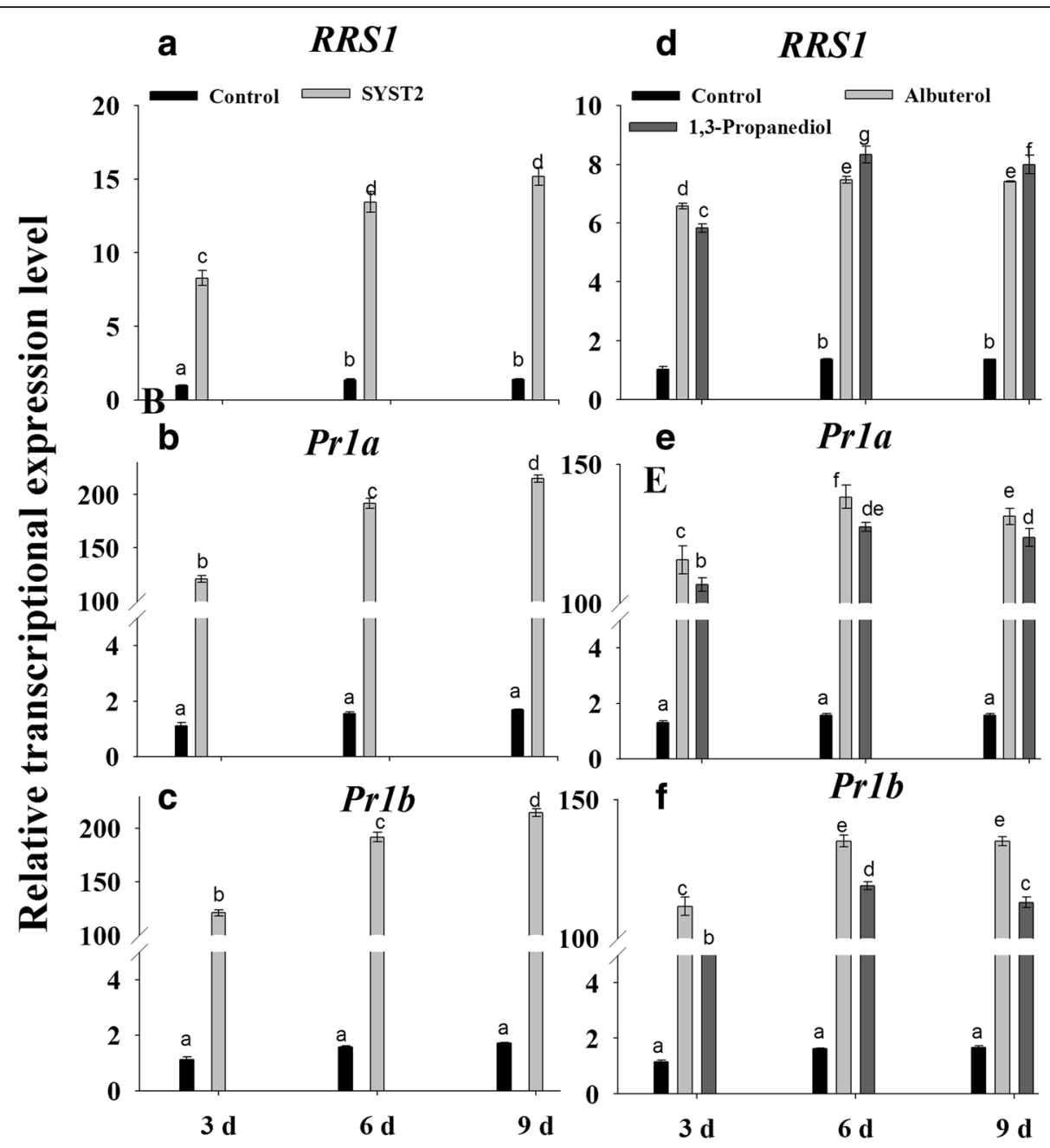

Fig. 9 Transcriptional expression profiles of genes involved in resistance after exposure to VOCs. QRT-PCR was performed with SYBR Green/Fluorescent qPCR master mix (Takara) on a Roche-480 system (Roche), taking EF-1a was as an internal reference. The relative expression levels of RRS1(a), Pr1a (b) and Prib (c) were determined after exposure to VOCs by SYST2 alone or in combination with Rs. Similarly, the relative expression levels of RRS1(d), Pria (e) and Prib (f) were determined after exposure to synthetic VOCs. Error bars indicate standard errors of the means. Different lower case letters above the error bars represent significant differences according to Duncan's multiple-range test $(P \leq 0.05)$ using SPSS software (SPSS, Chicago, IL)

induce plant growth in tomato. As we described above that SYST2-VOCs inhibited the growth of pathogen along with plant growth promoting activity. Although, Rs-VOCs also inhibited the growth of SYST2, but to a much lesser extent. In this context, SYST2-VOCs might be influenced in two positive ways: inhibiting pathogen growth and inducing systemic resistance, resulting in better plant growth. Our results suggested that SYST2VOCs can elicit plant defense mechanisms. Molecular studies revealed that, resistance occurs as a result of an increase in the concentration of metabolites and defense-related enzymes, including PAL. The enzyme PAL has a significant role in the regulation of lignin accumulation and the creation of defensive structures, besides with the production of phenols which act as chemical defenses $[37,38]$. PAL is also involved in plant salicylic-acid-mediated defense against plant pathogens while PPO catalyzes the oxygen-dependent oxidation of phenols. Both PPO and PAL participate in resistance mechanism against pathogenic microbes [39, 40]. Our results revealed an increase in both PPO and PAL in response to pathogen attack when plants were exposed to VOCs. These results are in conformity with previous results as a rise in the activities of PAL, PPO and PO enzymes was observed in Bacillus-treated tomato plants leading to the reduction of Fusarium wilt [41]. The results showed the up-regulation of transcriptional expression of $\mathrm{R}$ gene RRS1 along with the overexpression of Pr1a and Pr1b when exposed to VOCs after inoculation with Rs. The resistant gene RRS1 is specifically the bacterial wilt resistance gene in tobacco and the upregulation of RRS1 confirmed the activation of induced 
systemic resistance in tobacco [42]. The up-regulation of $\mathrm{R}$ gene (RRS1-R) in Col plants exhibited a clear increase in resistance level against wilt disease [43, 44]. Similarly, overexpression of $\mathrm{R}$ gene, $R E-b w$ in eggplant, resulting in increased resistance, proved that the $R E-b w$ was specifically a bacterial wilt resistant $\mathrm{R}$ gene. The upregulation of transcriptional expression of Prla and Pr1b suggested that SA signaling-path way, might be involved as PR1a is activated during SA-dependent signaling pathway. Our results suggest that ISR can be achieved without any contact between PGPRs and plants, demonstrating that VOCs might be involved usually in the process of induced systemic resistance $[45,46]$.

\section{Conclusion}

Our findings have shown that SYST2-VOCs significantly stimulated plant growth promotion and induced systemic resistance as well. Albuterol and 1,3-propanediol were found to be the key factors for growth promoting and ISR activity. SYST2-VOCs inhibited the growth of $R s$ and vice versa but inhibition of Rs by SYST2-VOCs, appeared to be much greater, resulting in the suppression of Rs inoculum level. Exposure to SYST2-VOCs or individual compounds altered the expression of genes involved in expansin, ethylene production, resistance and pathogenesis-related proteins.

\section{Methods}

\section{Bacterial strain, plant material, and growth conditions}

Tobacco (Nicotiana tabacum) seeds and both bacterial strains, Bacillus subtilis SYST2 (Accession no. GU568180.1), and Ralstonia solanacearum TBBS1 (Accession no. KY003096) used in this study, were obtained from our lab (Lab of biocontrol and bacterial molecular biology lab, Nanjing Agriculture University Nanjing). SYST2 was grown on Luria-Bertani (LB) medium at $37{ }^{\circ} \mathrm{C}$ overnight and stock cultures were maintained in LB broth supplemented with $30 \%$ glycerol at $-20{ }^{\circ} \mathrm{C}$. Ralstonia solanacearum TBBS1 was grown in tetrazolium chloride (TZC) agar medium (added $0.05 \%$ TTC) [47] for $48 \mathrm{~h}$ at $28{ }^{\circ} \mathrm{C}$. "The stock" of $R s$ was preserved in sterilized distilled water at room temperature. Single colonies with a pink center were transferred to Casamino Acid Peptone Glucose (CPG) agar medium for experimental use [48]. Tobacco seeds were surface-sterilized by soaking in $70 \%$ ethanol for $1 \mathrm{~min}$ in an Eppendorf, followed by soaking in sodium hypochlorite (30\%) for $15 \mathrm{~min}$, rinsed four to five times in sterile, distilled water and dried on filter paper. The sterilized seeds were placed on Petri plates containing half-strength Murashige and Skoog salt (MS) medium [49], having $0.8 \%$ agar and $1.5 \%$ sucrose with $5.7 \mathrm{pH}$ value.

\section{Growth-promoting activity of VOCs by SYST2 in vitro and} in planta

For in vitro assays, two types of experimental systems were designed; "divided plate system" and "two Petri plate system". For divided plate system, seven germinated seedlings were transplanted in one partition and bacteria were spot inoculated on the other partition. For "two plate system" we used soil as a medium for plant growth. A small plate $(160 \mathrm{~mm} \times 20 \mathrm{~mm})$ having minimal salt media for bacterial growth was placed in a large plate $(50 \mathrm{~mm} \times 15 \mathrm{~mm})$ having plant growth media $(40 \%$ organic matter mixed soil $+60 \%$ vermiculite). Seven equal size germinated seedlings were transplanted in the large plate containing soil mixture while bacteria were spot inoculated in the small plate. Plates were double sealed and incubated for further 10 days at $25{ }^{\circ} \mathrm{C}$ under a $12 \mathrm{~h}$ light $/ 12 \mathrm{~h}$ dark photoperiod. The effect of VOCs produced by SYST2 on plant growth was determined by evaluating the differences in fresh green and dry weights.

For in planta assay, SYST2 was inoculated on a Petri plate placed at the bottom of a tissue culture jar $(12 \times 10 \mathrm{~cm})$ as described by Park et al. (2015) [18]. Five germinated tobacco seedlings of equal size were transferred to the plastic pots $(6 \times 3 \mathrm{~cm})$ containing soil (appropriate amounts of sand, clay, and organic matter), fixed on the glass jars and sealed with Parafilm to avoid the escape of VOCs produced by bacterial strains (SYST2 or DH5 $\alpha$ ). Five or six small holes $(2 \mathrm{~mm})$ were made in the bottom of the pots to allow the roots to be exposed to the VOCs. The pots fixed on the jars were then placed at $28 / 22{ }^{\circ} \mathrm{C}$ day/night under 16/8-h light/ dark photoperiod. Plant growth enhancement was determined by evaluating the differences in fresh green weight, dry weight and leaf area between the controls and plants exposed to VOCs.

\section{Effect of volatiles produced by pathogenic bacteria on plant growth and efficiency of PGPR strain SYST2 in vitro and in planta}

For in vitro assay, we used three partitioned plates, having MS media in one partition for plant growth and minimal salt media in other two partitions for inoculation of PGPR strain SYST2 and pathogenic strain Rs. Five germinated seedlings were transplanted in one partition, $R s$ was spot inoculated in 2nd partition while SYST2 was spot inoculated in the third partition. One set of plates was inoculated with only SYST2 and one set with Rs only while sterilized water was used in the third partition. In control plates, only germinated seedlings were placed. Plates were sealed and incubated as described earlier and data was taken after two weeks. To evaluate the effect of VOCs produced by pathogenic bacteria on plant growth and efficiency of SYST2 in 
planta, we placed two Petri plates each inoculated with SYST2 or Rs in one jar. In other two jars, SYST2 or Rs was spot inoculated in Petri plates, placed individually in each jar while DH5 $\alpha$ or only water was used in controls. Five germinated tobacco seedlings of equal size were transplanted into the pots as described above and placed at $28 / 22{ }^{\circ} \mathrm{C}$ day/night under $16 / 8$-h light/dark photoperiod. Fresh green weight, dry weight, and leaf area were measured after 28 days.

GC-MS profile of VOCs produced by pathogenic bacteria $R s$ A 20- $\mu$ l suspension of overnight grown Rs cells was inoculated to $30 \mathrm{ml}$ of MS agar medium in a $100-\mathrm{ml}$ vial at $28{ }^{\circ} \mathrm{C}$. A 2-cm divinyl benzene/carboxen /PDMS (DCP, 50/30 $\mu \mathrm{m}$ ) solid phase microextraction (SPME) fiber (Supelco, Bellefonta, PA, USA) was used for collection of VOCs. After three days, the SPME fiber was inserted into the headspace of the vial containing bacteria and incubated at $50{ }^{\circ} \mathrm{C}$ for $30 \mathrm{~min}$. The GC-MS analysis was performed using a Bruker 450-GC gas chromatograph complemented with a Bruker 320-MS mass spectrometer as described by [50]. Helium gas was used as the carrier at a flow rate of $1 \mathrm{ml} \mathrm{min}^{-1}$. The SPME fiber was desorbed at $220^{\circ} \mathrm{C}$ for $5 \mathrm{~min}$, and GC-MS was run for $25 \mathrm{~min}$. The starting temperature of the column was $35{ }^{\circ} \mathrm{C}$ for $3 \mathrm{~min}$, which was increased to $180{ }^{\circ} \mathrm{C}$ at a rate of $10{ }^{\circ} \mathrm{C} / \mathrm{min}$, further increased to $240{ }^{\circ} \mathrm{C}$ at $4{ }^{\circ} \mathrm{C} / \mathrm{min}$, and then held for $5 \mathrm{~min}$. The mass spectrometer was operated in the electron ionization mode at $70 \mathrm{eV}$ with a source temperature of $220{ }^{\circ} \mathrm{C}$, with continuous scanning from $50 \mathrm{~m} / \mathrm{z}$ to $500 \mathrm{~m} / \mathrm{z}$. The data in the NIST/EPA/NIH Mass Spectrum Library (Agilent Technologies, Santa Clara, CA, USA) was used for comparison and analysis to identify the compounds [10].

\section{Effect of VOCs produced by Rs on the growth of PGPR strain SYST2}

To evaluate the effect of Rs-VOCs on the growth of SYST2, I-plate system was used which consisted of centrally partitioned plastic Petri dishes $(85 \times 15 \mathrm{~mm})$ with no physical contact between the two microorganisms grown on either side. Ten $\mu \mathrm{l}$ of (18-24 h) Rs culture $\left(1 \times 10^{7} \mathrm{CFU} \mathrm{ml}{ }^{-1}\right)$ grown in CPG broth was dropped in one partition while $10 \mu \mathrm{l}$ of liquid overnight-grown culture of SYST2 $\left(1 \times 10^{8} \mathrm{CFU} \mathrm{ml}{ }^{-1}\right)$ was dropped in the other partition containing modified minimal salt medium (MS) (1.5\% agar, 1.5\% sucrose, and $0.4 \%$ TSA $(w / v))$. The plates were double sealed and incubated at $28{ }^{\circ} \mathrm{C}$ for five days. Only pathogen and only SYST 2 were inoculated on control plates. The diameter of $R s$ and SYST2 were measured in $\mathrm{mm}$ and viable cells were also counted by the 10 -fold serial dilution technique. Each experiment was performed with five replicates, and the experiment was repeated three times.

\section{Effect of VOCs produced by SYST2 on activation of induced of systemic resistance in tobacco against bacterial wilt}

To investigate the effect of VOCs on wilt disease development, the experiment was conducted both in "two plate system" and in plastic pots fitted on jars. Seven germinated tobacco seedlings were dipped in the suspension of $R s\left(1 \times 10^{7} \mathrm{CFU} / \mathrm{ml}\right)$ cells and then transplanted into the large plate containing soil mixture while A $20-\mu \mathrm{l}\left(10^{8} \mathrm{CFU} / \mathrm{ml}\right)$ suspension of overnight grown PGPR strain SYST2 or synthetic chemical (albuterol and 1,3-propnaediole) was dropped into the small plate. The VOCs Albuterol and 1,3-propnaediole were identified from bacillus strain SYST2 using GC-MS analysis, in our previous experiment, and were proved as significant plant growth promoting VOCs [11]. For individual VOC evaluation, $50 \mu \mathrm{l}$ of each chemical with two different concentrations, i.e., $1 \mathrm{mM}$ albuterol, $0.1 \mathrm{mM}$ albuterol $10 \mathrm{mM}$ 1,3-propanediol and $1 \mathrm{mM}$ 1,3-propanediol were used in one compartment, while in other compartment, tobacco seedlings were grown as described earlier. After one week of exposure to VOCs, wilt symptoms were observed and the data were recorded using the formula below. For in planta assay, plastic pots were fixed on glass jars $(60 \times 120 \mathrm{~mm})$ and sealed with Parafilm to avoid the escape of VOCs from the jar, and a Petri plate $(35 \times 12 \mathrm{~mm})$ was placed at the bottom of the jar. Five or six small holes $(2 \mathrm{~mm})$ were made in the bottom of the pots to allow the roots to be exposed to the VOCs. Five germinated tobacco seedlings were transplanted in each pot. After 1 week of plant growth, SYST2 or DH5 $\alpha$ $\left(20 \mu \mathrm{l}, 10^{-7} \mathrm{cfu} / \mathrm{ml}\right)$ each was spot inoculated on Petri plates. DH $5 \alpha$ and sterilized water were used as a control. To test the effect of synthetic VOCs in pot experiment, $100 \mu \mathrm{l}$ of each compound with two different concentrations, i.e., $1 \mathrm{mM}$ albuterol, $0.1 \mathrm{mM}$ albuterol $10 \mathrm{mM}$ 1,3-propanediol and $1 \mathrm{mM} \mathrm{1,3-propanediol} \mathrm{were} \mathrm{applied}$ to small plates, that were placed at the bottom of jars. All pots were inoculated with a suspension of $R s$ (at an OD of 0.1 at $600 \mathrm{~nm}$ ) by dipping the roots in the suspension, except the non-inoculated control, and then the seedlings were re-planted in the pots. The pots were placed in a growth chamber at $28 / 22{ }^{\circ} \mathrm{C}$ day/night temperature for a $16 / 8$-h light/dark photoperiod at $85 \%$ relative humidity. The data for wilt was collected after 21 days of exposure to bacterial VOCs or synthetic VOCs, using the following formula: Disease index $(\%)=[\Sigma(n i \times v i) \div(V \times N)] \times 100$, where $n i$ indicates the number of plants with the respective disease rating; $v i=$ disease rating; $V=$ the highest disease rating (5) and $N=$ the number of plants observed. The disease rating was calculated using the scale: $1=$ no symptoms, $2=$ one leaf wilted, $3=$ two to three leaves wilted, $4=$ four or more leaves wilted and $5=$ whole plant 
wilted. The experiment was performed using a completely randomized design with five replicates, and the whole experiment was repeated thrice.

\section{Polyphenol Oxidase (PPO) and Phenylalnine ammonia Lyases (PAL) activity}

Five germinated tobacco seedlings were transplanted in each pot. After 1 week of plant growth, SYST2 (20 $\mu \mathrm{l}$, $10^{-7} \mathrm{cfu} / \mathrm{ml}$ ) was spot inoculated on Petri plates. DH5 $\alpha$ and sterilized water were used as a control. After 10 days of VOCs exposure we inoculated a set of plants with $R s$ while control plants were inoculated with sterilized water only. Leaf samples were collected 0 (pre-inoculation), 24, 48, 72, 48, 72, 96 and $120 \mathrm{~h}$ after inoculation of Rs. Samples were stored directly in liquid nitrogen prior to analysis. For PPO extracts, $1.0 \mathrm{~g}$ of leaf tissue samples were taken, homogenized in $5 \mathrm{~mL}$ of phosphoric acid extracting buffer $(0.05 \mathrm{M}$ phosphate, $\mathrm{pH}$ 7.0), filtered and centrifuged at $12,000 \mathrm{rpm}$ for $20 \mathrm{~min}$. The whole process was performed at $4{ }^{\circ} \mathrm{C}$ using ice and the supernatants were collected for enzyme activity analyses. A similar extraction process was used for PAL extraction except for the buffer solution (0.05 M boric acid, $5 \mathrm{mM}$ mercaptoethanol, $1 \mathrm{mM}$ EDTA and $0.05 \mathrm{~g}$ of polyvinylpyrrolidone). The enzyme activity was evaluated as described by Li [51].

\section{Effect of SYST2-VOCs on transcription of genes involved in growth regulation and systemic resistance}

Total RNA was extracted from leaf samples using TRIzol reagent (Invitrogen Biotechnology Co., Carlsbad, CA, U.S.A.) according to the manufacturer's instructions. To determine the effect of SYST2-VOCs on transcription of genes involved in growth regulation, samples were taken at 7,14 and 21 days exposure to VOCs while to determine the effect of VOCs on transcription of resistance related genes, samples were taken at 3, 6 and 9 days after inoculation of the pathogen. First-strand cDNA was synthesized using reverse transcriptase (TaKaRa Bio Inc., Tokyo, Japan) and random hexamer primers. Real-time PCR was performed using SYBR Green/Fluorescent qPCR master mix (Takara) on a Roche-480 system (Roche) using the $E F-1 \alpha$ gene [52] as an internal reference. The transcriptional expression levels of resistance related genes RRS1,Pr1a and Pr1b1 while growth regulation-related genes (NtEXPA1, NtEXPA2, and ACO1) were detected. The qRT-PCR program consisted of denaturation at $95^{\circ} \mathrm{C}$ for $1 \mathrm{~min}$, followed by 40 amplification cycles at $95{ }^{\circ} \mathrm{C}$ for $5 \mathrm{~s}, 57{ }^{\circ} \mathrm{C}$ for $30 \mathrm{~s}$, and $72{ }^{\circ} \mathrm{C}$ for $30 \mathrm{~s}$. The specific primers used in this study are listed in Additional file 1: Table S1. Each sample was replicated thrice for $\mathrm{qPCR}$, and $2-\Delta \Delta \mathrm{Ct}$ method was used to analyze gene expression level [53].

\section{Statistical analysis}

To evaluate the significance of the treatments, the data from each experiment was analyzed using analysis of variance (ANOVA) and Duncan's multiple-range test was employed to assess differences among treatments at $P=0.05$ using SPSS ver. 17.0 statistical software (SPSS, Chicago, IL). Graphs and figures were plotted using sigma plot version 10.0.

\section{Additional file}

Additional file 1: Primers used in this study and VOCs produced by Ralstonia solanacearum. (DOCX $34 \mathrm{~kb}$ )

\section{Abbreviations \\ CPG: Casamino Acid Peptone Glucose; DOVE-MO: Data of volatiles emitted by microorganisms; ISR: Induced the systemic resistance; LB: Luria-Bertani; MS: Murashige and Skoog; PAL: Phenylalnine Ammonia Lyases; PGPR: Plant growth-promoting rhizobacteria; PPO: Polyphenol Oxidase; Rs: Ralstonia solanacearum TBBS1; SPME: Solid phase microextraction; TZC: Tetrazolium chloride; VOCs: Volatile organic compounds}

\section{Acknowledgments}

We are thankful Dr. Muhammad Abid for technical assistance regarding critical review of manuscript.

\section{Funding}

This work was supported by the grants from the National key R\&D Program of China (2017YFD0200400), National Natural Science Foundation of China (31471811), the Agro-scientific Research in the Public Interest (20130315), the National Natural Science Foundation of China (31601589) and the Key research and development program of Jiangsu Province (BE2015354).

\section{Availability of data and materials}

The datasets used and/or analyzed during the current study are available from the corresponding author on reasonable request.

\section{Author's contribution}

HAST, QG, HW and XG conceived and designed the experiments. HAST performed most of the experiments. AS and FR performed the quantitative real time-PCR and WR recorded photosynthesis rate in tobacco. ZH performed the enzyme analysis. QG, AS, and FR analyzed the data. HAST, WR and ZH wrote the manuscript. All authors read and approved the final manuscript.

Ethics approval and consent to participate

Not applicable.

\section{Consent for publication}

Not applicable.

\section{Competing interests}

The authors declare that they have no competing interests.

\section{Publisher's Note}

Springer Nature remains neutral with regard to jurisdictional claims in published maps and institutional affiliations.

\section{Author details}

'Department of Plant Pathology, College of Plant Protection, Nanjing Agricultural University, Key Laboratory of Integrated Management of Crop Diseases and Pests, Ministry of Education, Weigang No.1, Nanjing 210095, People's Republic of China. ${ }^{2}$ Plant Pathology section, Tobacco Research station, Pakistan Tobacco Board, Ministry of Commerce, Govt. of Pakistan, Hayatabad, Peshawar, Pakistan. 


\section{Received: 16 February 2017 Accepted: 23 July 2017}

\section{Published online: 02 August 2017}

\section{References}

1. Hayward AC. Biology and epidemiology of bacterial wilt caused by Pseudomonas solanacearum. Annu Rev Phytopathol. 1991:65-87.

2. Peeters N, Guidot A, Vailleau F, Valls M. Ralstonia solanacearum, a widespread bacterial plant pathogen in the post-genomic era. Mol Plant Pathol. 2013;14:651-62.

3. Xue QY, Chen Y, Li SM, Chen LF, Ding GC, Guo DW, et al. Evaluation of the strains of Acinetobacter and Enterobacter as potential biocontrol agents against Ralstonia wilt of tomato. Biol Control. 2009:48:252-8.

4. Yunus FN, labal M, Jabeen K, Kanwal Z, Rashid F. Antagonistic activity of Pseudomonas fluorescens against fungal plant pathogen Aspergillus niger. Sci Lett. 2016:4:66-70.

5. Xie S, Wu H, Zang H, Wu L, Zhu Q, Gao X. Plant growth promotion by Spermidine-producing Bacillus subtilis OKB105. MPMI. 2014;27:655-63.

6. Punja ZK, Utkhede RS. Using fungi and yeasts to manage vegetable crop diseases. Trends Biotechnol. 2003;21:400-7.

7. Idriss EE, Makarewicz O, Farouk A, Rosner K, Greiner R, Bochow H, et al. Extracellular phytase activity of bacillus amyloliquefaciens FZB45 contributes to its plant-growth-promoting effect. Microbiology. 2002;148:2097-109.

8. Wu L, Wu H, Chen L, Xie S, Zang H, Borriss R, et al. Bacilysin from Bacillus amyloliquefaciens FZB42 has specific bactericidal activity against harmful algal bloom species. Appl Environ Microbiol. 2014;80:7512-20.

9. Raza W, Ling N, Liu D, Wei Z, Huang Q, Shen Q. Volatile organic compounds produced by Pseudomonas Fluorescens WR-1 restrict the growth and virulence traits of Ralstonia solanacearum. Microbiol Res. 2016;192:103-13.

10. Raza W, Ling N, Yang L, Huang Q, Shen Q. Response of tomato wilt pathogen Ralstonia solanacearum to the volatile organic compounds produced by a biocontrol strain Bacillus amyloliquefaciens SQR-9. Sci Rep. 2016;6:24856.

11. Tahir HAS, Gu Q, Wu H, Raza W, Hanif A, Wu L. Plant growth promotion by volatile organic compounds produced by Bacillus subtilis SYST2. Front Mol Biol. 2017:8:1-11.

12. Tahir HAS, Gu Q, Wu H, Niu Y, Huo R, Gao X. Bacillus volatiles adversely affect the physiology and ultra-structure of Ralstonia solanacearum and induce systemic resistance in tobacco against bacterial wilt. Sci Rep. 2017:7:40481.

13. Raza W, Yousaf S, Rajer FU. Plant growth promoting activity of volatile organic compounds produced by biocontrol strains. Sci Lett. 2016:4:40-3.

14. Wheatley RE. The consequences of volatile organic compound mediated bacterial and fungal interactions. Anton Leeuw J Microb. 2002;81:357-64.

15. Ryu C, Farag MA, Hu C, Reddy MS, Wei H, Pare PW, et al. Bacterial volatiles promote growth in Arabidopsis. PNAS. 2003;100:4927-32.

16. Zou C, Li Z, Yu D. Bacillus megaterium strain XTBG34 promotes plant growth by producing 2-pentylfuran. J Microbiol. 2010;48:460-6.

17. Lee B, Farag MA, Park HB, Kloepper JW, Lee SH, Ryu CM. Induced resistance by a long-chain bacterial volatile: elicitation of plant systemic defense by a C13 volatile produced by Paenibacillus polymyxa. PLoS One. 2012:7:e48744.

18. Park YS, Dutta S, Ann M, Raaijmakers JM, Park K. Promotion of plant growth by Pseudomonas Fluorescens strain SS101 via novel volatile organic compounds. Biochem Biophys Res Commun. 2015;461:361-5.

19. Lemfack MC, Nickel J, Dunkel M, Preissner R, Piechulla B. mVOC: a database of microbial volatiles. Nucleic Acids Res. 2014:42:744-8.

20. Bailly A, Weisskopf L. Current knowledge and future challenges. Plant Signal Behav. 2012:7(1):1-7.

21. Kai M, Piechulla B. Impact of volatiles of the rhizobacteria Serratia odorifera on the moss Physcomitrella patens. Plant Signal Behav. 2014;5:444-6.

22. Ryu C, Farag MA, Hu C, Reddy MS, Kloepper JW, Pare PW. Bacterial volatiles induce systemic resistance in Arabidopsis. Plant Physiol. 2004;134:1017-26.

23. Song GC, Ryu CM. Two volatile organic compounds trigger plant selfdefense against a bacterial pathogen and a sucking insect in cucumber under open field conditions. Int J Mol Sci. 2013;14:9803-19.

24. Zhang H, Kim M-S, Krishnamachari V, Payton P, Sun Y, Grimson M, et al. Rhizobacterial volatile emissions regulate auxin homeostasis and cell expansion in Arabidopsis. Planta. 2007;226:839-51.

25. Ortíz-Castro R, Contreras-Cornejo HA, Macías-Rodríguez L, López-Bucio J. The role of microbial signals in plant growth and development. Plant Signal Behav. 2014:4:701-12.

26. Xie $X$, Zhang $H$, Pare P. Sustained growth promotion in Arabidopsis with long-term exposure to the beneficial soil bacterium Bacillus subtilis (GB03). Plant signal. Behav. 2009;4:948-53. 27
27. Sang Y, Ryu KC, Lee $S$, Bee $H$, Soo K, Jung $H$, et al. Proteome analysis of Arabidopsis seedlings exposed to bacterial volatiles. Planta. 2010;1370:1355-1370

28. Francis I, Holsters M, Vereecke D. The gram-positive side of plant-microbe interactions. Environ Microbiol. 2010;12:1-12.

29. Gu Y-Q, Mo M-H, Zhou J-P, Zou C-S, Zhang K-Q. Evaluation and identification of potential organic nematicidal volatiles from soil bacteria. Soil Biol Biochem. 2007;39:2567-75.

30. Weisskopf $\mathrm{L}$. The potential of bacterial volatiles for crop protection against phytophathogenic fungi. 2013;1352-63.

31. Wu Y, Zhou Y, Song J, Hu X, Ding Y, Zhang Z. Using green and red fluorescent proteins to teach protein expression, purification, and crystallization. Biochem Mol Biol Educ. 2008:36:43-54

32. Zhang H, Xie X, Kim M-S. Kornyeyev D a, Holaday S, Paré PW. Soil bacteria augment Arabidopsis photosynthesis by decreasing glucose sensing and abscisic acid levels in planta. Plant J. 2008;56:264-73.

33. Wang S., Wu H., Qiao J., Ma L., Liu J., Xia G., and Gao X. Molecular mechanism of plant growth promotion and induced systemic resistance to Tobacco Mosaic Virus by Bacillus spp. J. Microbiol. Biotechnol. 2009; 10. 4014/jmb.0901.0008.

34. Minerdi D, Bossi S, Gullino ML, Garibaldi A. Volatile organic compounds: a potential direct long-distance mechanism for antagonistic action of Fusarium oxysporum strain MSA 35. Environ Microbiol. 2009;11:844-54.

35. Choi D, Lee $\mathrm{Y}, \mathrm{Cho} \mathrm{H}-\mathrm{T}$, Kende $\mathrm{H}$. Regulation of expansin gene expression affects growth and development in transgenic rice plants. Plant Cell. 2003;15:1386-98.

36. Kwon YS, Ryu CM, Lee S. Proteome analysis of Arabidopsis seedlings exposed to bacterial volatiles. Planta. 2010:6:1355-70

37. Xu, L., Zhu, L., Tu, L., Liu, L., Yuan,D., Jin, L., et al. Lignin metabolism has a central role in the resistance of cotton to the wilt fungus Verticillium dahliae as revealed by RNA-Seq-dependent transcriptional analysis and histochemistry. J Exp Bot 2011:62:5607-5621.

38. Gayoso C, Pomar F, NovoUzal E, Merino F, llárduya ÓM. The Ve-mediated resistance response of the tomato to Verticillium dahliae involves $\mathrm{H}_{2} \mathrm{O} 2$, peroxidase and lignins and drives PAL gene expression. BMC Plant Biol. 2010;10:232-50

39. Mittler R, Vanderauwera S, Gollery S, Breusegem V. Reactive oxygen gene network of plants. Trends Plant Sci. 2004:9:490-8.

40. Finger A. In-vitro studies on the effect of polyphenol oxidase and peroxidase on the formation of polyphenolic black tea constituents. J Sci Food Agr. 1994;66:293-305.

41. Akram W, Anjum T. Quantitative changes in defense system of tomato induced by two strains of bacillus against Fusarium wilt. Indian J Fund Appl Life Sci. 2011;1:7-13.

42. Lei L, Long R, Yiliang Q, Zhichao W, Yang H, Xueyong $\mathrm{S}$, et al. Detection on R R S 1 gene of bacterial wilt resistance from Arabidopsis in tobacco for homolog. Chinese Agric Sci Bull. 2012;28:137-40.

43. Hirsch J, Feng DX, Bittner-eddy P, Deslandes L, Olivier J, Beynon J, et al. Resistance to Ralstonia solanacearum in Arabidopsis thaliana is conferred by the recessive RRS1-R gene. PNAS. 2002;99:2404-9.

44. Narusaka M, Shirasu K, Noutoshi Y, Kubo Y, Shiraishi T, Iwabuchi M, et al. RRS1 and RPS4 provide a dual resistance-gene system against fungal and bacterial pathogens. Plant J. 2009:60:218-26.

45. Van Loon LC GB. Increased plant fitness by rhizobacteria. In: H S, editor. Mol. Ecotoxicol. plants. Molecular. Berlin: Springer; 2004;177-205.

46. Van De MJE, De VRCH, Dekkers E, Pineda A, Guillod L, Bouwmeester K, et al. Metabolic and transcriptomic changes induced in arabidopsis by the rhizobacterium Pseudomonas. Plant Physiol. 2012;160:2173-88.

47. Kelman A. The relationship of pathogenicity in Pseudomonas solanacearum to colony appearance on a tetrazolium chloride medium. Phytopathology. 1954;44:693-5.

48. Hendrickt CA, Sequeira L. Lipopolysaccharide-defective mutants of the wilt pathogen Pseudomonas solanacearum. Appl Environ Microbiol. 1984;48:94-101.

49. Murashige T, Skoog F. A revised medium for rapid growth and bioassays with tobacco tissue cultures. Physiol Plant. 1962;15:473-97.

50. Farag MA, Ryu CM, Sumner LW, Paré PW. GC-MS SPME profiling of rhizobacterial volatiles reveals prospective inducers of growth promotion and induced systemic resistance in plants. Phytochemistry. 2006:67:2262-8.

51. Li SM, Hua GG, Liu HXGJ. Analysis of defence enzymes induced by antagonistic bacterium Bacillus subtilis strain AR12 towards Ralstonia solanacearum in tomato. Ann Microbiol. 2008;58:573-8. 
52. Berberich T, Sugawara K, Harada M, Kusano T. Short communication molecular cloning, characterization and expression of an elongation factor I a gene in maize. Plant Mol Biol. 1995;45407:611-5.

53. Livak KJ, Schmittgen TD. Analysis of relative gene expression data using real-time quantitative $P C R$ and the 2(-Delta Delta $C(T))$ method. Methods. 2001;25:402-8

Submit your next manuscript to BioMed Central and we will help you at every step:

- We accept pre-submission inquiries

- Our selector tool helps you to find the most relevant journal

- We provide round the clock customer support

- Convenient online submission

- Thorough peer review

- Inclusion in PubMed and all major indexing services

- Maximum visibility for your research

Submit your manuscript at www.biomedcentral.com/submit 